\title{
Religiosität als erwünschte Anregung in der Hermeneutik
}

\author{
Religiosity as Desired Stimulation in the Hermeneutics
}

\begin{abstract}
Paul Ricoeur insists on some kind of philosophical agnosticism although in everyday practice he is consciously bound to Christianity. That said, his poetical approach in Biblical hermeneutics when he confronts his position with his no believing contemporaries, very clearly shows that religious dimension of man, especially in its openness to new meanings, makes the understanding more prolific. This opens the way for new attempts of repeated search for human need for religious dimension also at Luc Ferry and Gianni Vattimo. It is not necessary that there is a very certain form of religiosity; the important thing is openness for these questions. In this way we liberate the science, to seriously confront with religious dimension of man. This is especially important in a globalized world where religiosity on one hand can be a cause for the clash of civilizations and on the other it can be a possibility of a common search for a world ethos.

\section{Keywords}

Poetics, hermeneutics, Paul Ricoeur, Luc Ferry, Gianni Vattimo, globalization, religiosity, origin of meanings.
\end{abstract}

\section{Einleitung}

Eine prinzipielle Auseinandersetzung zwischen der Wissenschaft und der Religion ist die Folge eines im Voraus vorbereiteten Verstehens der Realität und der Art und Weise des Zugangs zu dieser Realität. Die biblische Auffassung, dass alles von Gott und für Gott geschaffen ist, war die Grundlage der theologischen 
Deutung und des Verständnisses der Schöpfung des Einzelnen. Die Schöpfung ist der Abglanz des Schöpfers. Mit dem Christentum kommt das noch stärker zur Geltung, da diesem Aspekt zusätzlich ein anthropologischer Zug hinzukam. Christus wurde Mensch. Durch diesen Menschen, durch seine eigene körperliche und geistige Natur offenbarte er Gott in seiner Vollkommenheit. Deswegen sorgte die Theologie noch stärker für das richtige Verständnis des Menschen und die daraus folgende Beziehung zu ihm. Descartes Versuch, die Zuständigkeitsbereiche Gottes und des Menschen voneinander zu trennen, ermöglichte der Wissenschaft einigermaßen einen freieren Weg. In der Physik, Astronomie und anderen technischen Wissenschaften konnte sich der Mensch immer auf die Tatsache berufen, dass er durch seine wissenschaftliche Arbeit Gottes wunderbare Schöpfung näher kennen lernen will. Im Humanismus war das aber viel schwieriger. Jede Abweichung von der biblischen und von der offiziellen Deutung der Kirchen war gefährlich. Auch wenn es mehr oder weniger akzeptiert wurde, dass die Bibel keine technischen und naturwissenschaftlichen Erkenntnisse erörtert, konnte man das für das Verstehen des Menschen und für seine Rolle in der Welt nicht in Anspruch nehmen. Die Heilige Schrift, eigentlich jede Religion, erklärt vor allem den Menschen, seine Rolle in der Welt, und durch ihre Lehre entwirft sie dem Menschen eine gewisse Lebensweise. Dieses Feld teilt die Religion nicht nur mit der Philosophie, sondern mit einer ganzen Reihe humanistischer Wissenschaften. Eben diese liegen am häufigsten im Streit mit einer bestimmten Religion. Wenn wir uns nur auf die Buchreligionen beschränken, so können wir sagen, dass ihr Gottes- und Glaubensverständnis vor allem aus ihrem hermeneutischen Zutritt zu den Grundtexten hervorgeht. Die Deutung des heiligen Textes bestimmt das Verstehen des Glaubensinhalts. Die Hermeneutik wurde im 20. Jh. zum grundlegenden Philosophiezweig, der nie verheimlichte, dass er sich vorwiegend aus dem biblischen entwickelte. Heidegger in seiner Kritik an der Technik vermisst eben einen freien Zutritt des Menschen, der sich verstehen würde und nicht bloß ein Teil der determinierten technischen Welt wäre. Auch Vattimos Verstehen der Säkularisierung ruft nach einer neuen Hermeneutik, die im schwachen Denken den Glauben an Gott zulassen würde. Die Hermeneutik braucht trotz allem ein System, einen Hintergrund und zuletzt eine Offenheit, die immer wieder die Möglichkeit einer schöpferischen Interpretation bietet. Ohne diese kommt man zum Technizismus im strengen Sinne des Wortes: man wiederholt und dreht eine und dieselbe Bedeutung. Das ist aber nicht menschlich, noch weniger ist es im Interesse der Philosophie und letztendlich auch nicht im Interesse der Wissenschaft, die immer wieder nach neuen Entdeckungen strebt. Vattimos Äußerung auf die 
Frage seines Freundes über den Glauben lautete: „Ich antworte, ich glaubte, dass ich glaube“ ${ }^{\text {, }}$, was im Original auch der Titel seines Werkes ist, ist keine seltene Ausnahme des zeitgenössischen philosophischen Denkens, besonders wenn es um ethisch-gesellschaftliche Fragen geht. Habermas schließt sein Werk »Glauben und Wissen« so ab: „Dass der Gott, der die Liebe ist, in Adam und Eva freie Wesen schafft, die ihm gleichen, muss man nicht glauben, um zu verstehen, was mit der Ebenbildlichkeit gemeint ist. Liebe kann man ohne Erkenntnis in einem anderen, Freiheit ohne gegenseitige Anerkennung nicht geben. Dieses Gegenüber im Menschengestalt muss seinerseits frei sein, um die Zuwendung Gottes erwidern zu können. Trotz seiner Ebenbildlichkeit wird freilich auch dieser Andere als Geschöpf Gottes vorgestellt. Hinsichtlich seiner Herkunft kann er Gott nicht ebenbürtig sein. Diese Geschöpflichkeit des Ebenbildes drückt eine Intuition aus, die in unserem Zusammenhang auch dem religiös Unmusikalischen etwas sagen kann“'2. Gerade diese Bemühung um das Verstehen des Menschen und der Ethik ist diejenige, die sowohl die Theologie, wie auch die Philosophie und auch alle anderen humanistischen Disziplinen immer mehr vereinigt.

\section{Mensch - Gott}

Luc Ferry beginnt sein Werk Mensch - Gott ${ }^{3}$ mit der Feststellung, dass beim modernen Menschen die Frage nach dem Sinn des Lebens fehlt. Der Mensch, wenn er bewusst handelt, handelt immer auf Grund eines bestimmten Zieles. Das sollte zur Folge haben, dass in die Überlegung der zeitgenössischen Gesellschaft die Frage nach dem Tod nicht eingeschlossen werden kann. Es ist zwar tatsächlich so, dass Freud das Nachdenken über den Tod oft als krankhaft definiert, doch der Ausgangspunkt jeder ernsthaften philosophischen Auseinandersetzung ist eben die eigene Vergänglichkeit. Auch in den Philosophien, die eine bestimmte therapeutische Rolle übernehmen wollen, ist der Tod kein Tabu. Die Angst, die aus dem Bewusstsein über den Tod hervorgeht, ist kein Hindernis mehr, sondern ein schöpferischer Ausgangspunkt: „Wo das pathologische Verständnis der Angst an seine Grenzen stößt, gewinnt die tragische Auffassung an Plausibilität: Angst ist für sie der profunde Einblick in den Aufbau und die Zusammensetzung von Wirklichkeit und Welt, in das untergründige Sein, das gegenüber allem

\footnotetext{
${ }^{1}$ G. Vattimo, Glauben - Philosophieren, Stuttgart 1997, S. 76.

2 J. Habermas, Glauben und Wissen, Frankfurt am Main 2001, S. 30.

${ }^{3}$ Vgl. L. Ferry, L'Homme-Dieu, Paris 1996.
} 


\section{The Person and the Challenges \\ 130

vordergründigen Schein faszinierend und erschütternd in seiner Nacktheit ist. Alles wird zu nichts in der Angst: das Selbst, seine Beziehung zu anderen, die Welt als Ganzes - aber gerade dadurch tritt hervor, was unter Sinn verstanden werden kann“44.

In der säkularisierten Welt muss sich der Mensch viele Ziele, Aufgaben setzen, ohne die vorausgehenden Antworten zu bekommen. Alte religiöse und andere gesellschaftliche Normen haben keine Kraft mehr, darum ist jede Entscheidung Widerspiegelung einer reflexen Handlung oder der eigenen Auswahl. Im Alltagsleben geht das noch irgendwie. Man bekommt mit seiner Ausbildung und den zeitgenössischen Trends eine bestimmte Orientierung, doch man ist nicht mehr imstande, den endgültigen Sinn zu bestimmen, warum man sich bestimmten Aufgaben stellt. Moderne Trends sind nämlich kurzfristig und geben keine endgültigen Antworten. Die Konsumgesellschaft basiert auf einem schnellen Wechsel der Nachfrage. Das bedeutet aber gleichzeitig, dass das Handeln nur noch im Dienste des augenblicklichen Bedarfs steht. Der Mensch wird immer mehr zum Gefangenen des Augenblicks, was sich in einem bestimmten modernen Buddhismus widerspiegelt, wenn es sich um eine intellektuelle Elite handelt ${ }^{5}$.

Der ganze Prozess der Säkularisierung, der Abwertung der alten Werte, hat auch Folgen für die Ethik. Wenn der Lebenssinn als Ganzes mit der Säkularisierung verschwunden ist, verschwindet gleichzeitig damit auch die Anregung zu einer Handlung, wo der Mensch über seine augenblicklichen Bedürfnisse hinausgehen würde. Etwas zu unternehmen, was augenblicklich nicht im eigenen Interesse liegt, fordert eine sinnvolle Antwort. Wenn einst die Gesellschaft, die Religion und Kultur bestimmte Werte anerzogen und vermittelt haben, die im Ganzen des gesellschaftlichen Diskurses heilig waren, halfen sie dem Einzelnen bei der Überwindung der Anstrengung, als man diese Werte im Leben konkretisieren musste. Etwas war in der Gesellschaft heilig und darum war es auch sinnvoll, sich dafür zu opfern, sei es Familie, Heimat, Gott oder ähnliches. Heute existiert diese Fähigkeit des Opferns nicht mehr, weil wir nichts mehr als heilig empfinden. Der Verlust des Heiligen, der die Frucht einer bestimmten Gesellschaft war, ist die Folge der Säkularisierung.

Man soll sich nicht über dieses Prozess beklagen, doch es lohnt sich, ihn zu reflektieren. Für Ferry ist die Säkularisierung so lange sinnvoll, bis sie nicht zum gänzlichen Verschwinden des Heiligen führt. Den Weg, vom vormodernen Konzept des Heiligen zum zeitgenössischen, sieht er in der christlichen Theologie.

\footnotetext{
${ }^{4}$ W. Schmid, Mit sich selbst befreundet sein, Frankfurt am Main 2004, S. 27.

${ }^{5}$ Vgl. L. Ferry, L'Homme-Dieu.
} 
Diese Theologie wird nicht mehr das Christliche vor die Ethik stellen, sondern wird ihren Platz als letzte Sinngebung des menschlichen Handelns finden. So wird die Beziehung zu Gott nicht mehr der Grund der Ethik, sondern ihre Ergänzung: „Sie gibt der Beachtung der Gesetze den Sinn, erteilt der Pflicht Hoffnung, Liebe der Hochachtung, die christliche Note dem jüdischen Element“6. Das alles in einer Zeit, wo wir von einer gewissen Säkularisierung auch auf dem Gebiet der Ethik sprechen. Seiner Meinung nach ist es heute diese Zeit, wo die Ethik des kategorischen Imperativs überwunden wurde. Der Mensch ist nicht mehr bereit, nur das zu tun, was er müsste, sondern er sucht immer mehr Authentizität. Er fragt sich, warum und wie er aus sich herauszukommen soll. Er ist nicht mehr bereit, sich zu opfern, wenn er selbst kein Bedürfnis danach hat. Er will Gründe finden, die ihm selbst nützen würden, wenn nichts anderes, soll ihm wenigstens Selbstbewusstsein einflössen, ihm bei der Gestaltung seiner Lebensorientierung helfen. Es geht also um eine Wendung zum Subjekt, das freilich in bestimmten Grenzen selbst entscheiden will, was es tun soll und was nicht. Aber es geht nicht um das Aufhören jeder Opferbereitschaft. Wenn wir uns z.B. die Umfragen der Beliebtheit in Frankreich ansehen, können wir feststellen, dass mehr oder weniger immer diejenigen an der Rangliste oben stehen, die das Sinnbild der selbstlosen Aufopferung sind. Ferry fügt hinzu, dass die Befragten auf die Frage, warum eben diese Gruppen gewählt wurden, bestimmt antworten würden, dass sie nicht die kopflose Aufopferung, sondern die persönliche Entscheidung der ausgewählten Individuen schätzen würden. Heute werden diejenigen geschätzt, die sich von sich aus den anderen, ihren Bedürfnissen widmen können, und das aus Liebe und nicht aus Pflicht tun. Es geht nicht um die Aufopferung im Namen Eines oder Etwas, sondern um eine Aufopferung aus eigener Entscheidung. Die Aufopferung, Schenkung schließt immer etwas ein, was über uns hinausgeht. Niemand wird bewundert, wenn er sich bloß um sein eigenes Überleben bemüht. Die oben angeführten Beispiele und viele humanitäre Organisationen, die in letzter Zeit Massen zum größeren persönlichen Einsatz motivieren, zeigen auf, dass das Gespür für das Transzendente, für etwas, was über den Menschen hinausgeht, nach wie vor besteht.

Ferrys Meinung nach sollte es sich dabei um den Übergang von der sog. vertikalen Transzendenz zur horizontalen Transzendenz handeln ${ }^{7}$. Mit den Beispielen aus dem alltäglichen Leben könnte man sagen, dass die Aufopferung für die Heimat oder für die Ideen einer Revolution als etwas

${ }^{6}$ Ebd., S. 46.

${ }^{7}$ Vgl. ebd., S. 93. »Cette sacralisation de l'humain comme tel suppose le passage de ce que l’on pourait nommer une 'transcendance verticale' à une 'transcendace horizontale' «. 
allgemein Bewundertes, heute kaum akzeptabel ist. Das könnte uns an die selbstmörderischen Terroristen erinnern. Es werden aber Leute bewundert, die sich selbst aufgeopfert haben, um den konkreten Menschen zu helfen. Ärzte ohne Grenzen, opferungsbereite Helfer, Eltern und man könnte noch weitere aufzählen, zeigen heute eine Veränderung des Horizonts vom Vertikalen zum Horizontalen. All das spiegelt sich in der immer größeren Solidarität mit der ganzen Menschheit. Es gehtum eine bestimmte Humanisierung der Transzendenz. Die Aufopferung gilt nicht mehr einem Gott, einer abstrakten Idee, der Heimat, sondern dem konkreten Menschen. Das Heilige tritt aus seiner Unbestimmtheit in die Welt unserer Wahrnehmung, die Welt meines Nächsten. „Es scheint mir sogar, dass es interessanter ist, über konkrete Liebe zu philosophieren, als über die Wohltätigkeit, von der niemand wirklich weiß, wie und was sie ist. Weil es bestimmt eine konkrete Liebe gibt, die unserem Leben den Sinn gibt“" ${ }^{\text {. Christus }}$ machte als erster darauf aufmerksam. Er offenbart durch den Menschen, der seine Hilfe braucht, den himmlischen Vater. Wenn wir heute auf den Vorrang der konkreten Liebe zum Menschen aufmerksam machen, handelt es sich um den Prozess der wiederholten Menschwerdung Gottes.

Auf der anderen Seite entspringt aus der Humanisierung Gottes auch ihr Gegensatz, der sich in der Vergöttlichung des Menschen manifestiert. Dadurch wird auch die Heiligkeit des eigenen Lebens verstärkt. All das zeigt sich in ganz konkreten Sachen. Wenn einst die Eheschließung aus Liebe, auf Grund eigener Entscheidung eine Seltenheit war, ist das heute normal. Man berücksichtigt den Willen des Einzelnen, die Entscheidung des Menschen wurde heilig. Dasselbe gilt für die Hochachtung der Kinder. Einmal war das Kind Eigentum, heute wurde es zu demjenigen, auf den man Rücksicht nehmen und den man von seinem Anfang an respektieren soll. Wenn in der Vergangenheit viele Väter nicht einmal wussten, wie viele Kinder sie haben, unter ihnen auch der große Humanist Montesquieu, so ist heute jedes Kind kostbar und wird auch gesetzlich als selbständiges Individuum geschützt. All das spricht für den Übergang von der vertikalen zur horizontalen Transzendenz. In dieser konkreten Verbundenheit von Gott und Mensch zeigt sich der Übergang von Don Juan zur Agapé. Es existiert noch immer der Egoismus und er ist auch der Ausgangspunkt, doch man kann nicht behaupten, dass es sich bei der Liebe zum Kind nur um Egoismus handelt. Noch weniger bei unserer Bewunderung der Menschen, die es vermögen, sich für andere zu opfern. Die Liebe, die imstande ist, aus dem abstrakten Gebot zur

8 A. Comte-Sponville und L. Ferry, La sagesse des Modernes, Paris 1996, S. 444. Interessanterweise ist auch Papst Benedikt XVI. zu Ähnlichem gekommen ist. In seiner Enzyklika Gott ist Liebe - Deus caritas est - spricht er gerade von der menschlichen Liebe - Eros als Ausgangspunkt zur Begegnung und Verstehen der Liebe Gottes. 
konkreten Situation überzugehen, vermag immer mehr alle einzuschließen, die uns ähnlich sind ${ }^{9}$.

Um nicht nur bei einer Sentimentalität zu bleiben, müssen wir auch die alte Kraft der Religion mit einbeziehen, die fähig war zu verbinden und eine Gemeinschaft zu bilden. Die Heiligung des Gemeinschaftsgefühls ist diejenige, die dem Menschen den Daseinssinn gibt, der nicht nur auf hier und jetzt beschränkt ist, sondern immer wieder über das Individuum hinausreicht. Der Humanismus kann nicht nur auf einem Niveau der augenblicklichen Reaktion bleiben, einer Gefühlsbeunruhigung, etwas wie eine Moral in Not, sondern muss eine Ökonomie herstellen, die fähig wird, dem Menschen in seiner ganzen Dimension des Heiligen einen Sinn zu geben. Insbesondere, da die Demokratie, die das Abbild der Vergöttlichung des Menschen ist, einen engagierten Einzelnen braucht, der fähig wird, über die Befangenheit in hier und jetzt hinauszugehen. Das wird möglich, wenn es gelingen wird, die Tugend in die Liebe, also Dike in Philia einzuwurzeln. Dem alttestamentlichen Recht muss man aufs neue die neutestamentliche Liebe einhauchen. Das alles ist aber nur bei der Berücksichtigung der Transzendenz möglich, auch die der gesellschaftlichen.

Als Folge davon, so stellt Ferry fest, hat der wissenschaftliche oder materialistische Atheismus sowohl für den Einzelnen als auch für die Gesellschaft keine Zukunft. Früher oder später müsste man zugeben, wenn alle Kriterien der Wissenschaft konsequent berücksichtigt werden, dass der Mensch nicht der Autor seiner Handlungen, seiner Ideen ist, sondern nur das Produkt zahlreicher Gesetzlichkeiten. Gleichzeitig relativieren sich in gewissem Maße die atheistischen Thesen selbst. Die Behauptung, dass der Mensch das Produkt dieser oder jener Kraftlinien, Gesetzlichkeiten, Vererbbarkeiten oder der gesellschaftlichen Umgebung sei, ist für sie eine Tatsache, die sie mit wissenschaftlichen Methoden zu beweisen versuchen. Dabei geht es um eine deutliche Ausschließung der Transzendenz, der Möglichkeit also, aus der Situation herauszutreten, was ihnen freilich unmöglich macht, eine objektive Meinung zu äußern. „Eben die Negierung der realen Person nimmt dem Materialismus die Möglichkeit, in der Fülle des Humanismus zu sein“10. Es zeigt sich die Notwendigkeit nach der Transzendenz, die dem Menschen die Möglichkeit geben würde, über sich zu urteilen. Darin ist schon sowohl Husserls Verlangen nach dem Hinausgehen über

${ }^{9}$ Vgl. L. Ferry, La sagesse des Modernes, Paris 1996, S. 122. »Tout indique, au contraire, que des transcendaces se reconstituent, d'abord dans la sphère des sentiments individuels, mais, sans doute, bien au-delà, à travers la prise en considération de l'humanité dans son ensemble«.

${ }^{10}$ Ebd., S. 171. 
den Psychologismus und Soziologismus, wie auch Heideggers Definition des Menschen als Ex-istenz mit eingeschlossen.

Ferry zählt fünf Gründe auf, die die Notwendigkeit der Fortsetzung der christlichen Religiosität im Sinne der Humanisierung Gottes bzw. der Vergöttlichung des Menschen aufzeigen. Der erste Grund ist die Liebe, welche die Transzendenz in sich selbst einschließt. Die Liebe ist auf den Anderen gerichtet, reicht über den Augenblick, sogar über den Tod hinaus und vermag auch die Wahrheit, Gerechtigkeit, das Schöne zum Gegenstand zu haben. All das schließt die alte religiöse Dimension der Transzendenz ein. Der zweite Grund liegt in der menschlichen Natur als eines denkenden Wesens. Er findet in sich die Fähigkeit, Werte zu stellen, welche die Welt um ihn herum übersteigen. Es geht nicht nur um die eingeborenen Ideen, um die a priori Kategorien, sondern vor allem um Werte, die das gemeinsame Leben und die Zukunft erhalten und lenken können. Der dritte Grund ist die Tatsache, dass nur die transzendenten Werte, diejenigen also, die nicht im augenblicklichen Bedürfnis des Einzelnen gefangen sind, eine beständige Gemeinschaft der Personen und nicht bloß eine Vereinigung der Individuen bilden. Eine Person ist fähig, aus sich selbst etwas zu schaffen, zum Beispiel die Deklaration der Menschenrechte zu schreiben und sie nicht nur anzunehmen oder abzuschreiben. Gleichzeitig muss aber an dem Menschen etwas Heiliges sein, wenn man will, dass er aufopferungswert, der Liebe wert ist. Im vierten Grund steckt eben dieser transzendentale Wert, den der Liebende in der geliebten Person erspürt.

Die Opferung schließt gleichzeitig auch das Überragen der Zeit in sich ein. Der Mensch verweist eben durch die Aufopferung darauf, dass es noch etwas gibt, was wertvoller als sein Leben ist. Das kann natürlich nur dann sinnvoll sein, wenn das Leben des Opfers heilig ist. Damit schließt er den fünften Grund ein, der besagt, dass der Mensch überragungsfähig ist auch in Bezug auf die Sterblichkeit, er vermag über sie hinauszuwachsen. Darauf deuten von den Pyramiden an weiter alle großen Schöpfungen, die mit der Kultur der Erinnerung verbunden sind, nicht zuletzt auch die Denkmäler verschiedenen Nationalhelden. Das Paradoxe ist dabei, dass dieser menschgewordene Gott in Zeit und Ort gefangen ist, dabei ragt er mit jedem menschlichen Handeln immer wieder hinaus, ins Transzendentale ${ }^{11}$.

Einen ähnlichen Weg zur Religiosität kann man auch in Vattimos Verständnis der Säkularisierung finden. Im modernen Prozess der Entgöttlichung, der Entfremdung vom Glauben kann man, seiner Meinung nach, ein genuin

${ }^{11}$ „Sans la résurrection, en effet, la contradiction entre l'amour et la mort est beaucoup plus grande, mais c'est justement ça le problème que nous lègue la sécularisation du christianisme« (A. Sponville und L. Ferry, S. 447). 
christliches Phänomen sehen. „Gott selbst ,säkularisiert sich’, darum bleibt die Säkularisierung die innere und logische Entwicklung der jüdisch-christlichen Offenbarung und sie ist ihr nicht fremd oder feindlich“"12. Es geht um einen Gott, der sich vollkommen opfert, sich schenkt: „Klarer gesagt: Was ich wiederentdecke, ist eine Lehre, die ihren Grundpfeiler in der kenosis Gottes hat, und damit im Heil, das als Auflösung des natürlich-gewaltsamen Sakralen verstanden wird;... " ${ }^{13}$ Christus wird erneut zum Menschen, vollkommen hilflos, um dem Menschen den Weg zu öffnen, sich selbst übertreffen zu können. „Die im Verlauf des Nihilismus niemals abzuschließende Unendlichkeit ist vielleicht nur dadurch begründet, dass die Liebe als letzter Sinn der Offenbarung keine wahre Letztheit hat; und sie ist auf der anderen Seite der Grund dafür, dass die Philosophie am Ende der Epoche der Metaphysik entdeckt, dass sie ihrer Grundlage, dem objektiv, vor den Augen der Vernunft Gegebenem nicht mehr glauben kann und dass sie (wozu sie auch, oder gerade, durch die christliche Tradition erzogen wurde) der Gewalt bewusst wird, die in jeder Letztheit, in jedem ersten Prinzip, das alles Weiterfragen zum Schweigen bringt, beschlossen ist “14. Das Ende der klassischen Metaphysik, die auch die Ursache dieser Gewalt ist, bietet dem neuen Verstehen des Christentums Platz. Christus ist ein Ereignis, seiner Meinung nach, ein Ereignis der Schwächung, die bei jedem Menschen in seiner Konkretheit vorkommt. Die Offenbarung setzt sich in der Säkularisierung fort, und dadurch wird eine neue Menschwerdung ermöglicht.

Bei den beiden Philosophen geht es um den Weg von einer beginnenden, irgendwie wissenschaftlichen Denkweise, die in den philosophisch logischen Rahmen eingespannt wurde, bis zum persönlichen Suchen, das nicht als klassisch verstandene Bekehrung zu deuten ist, sondern sich, wegen der vorhergehenden Denkweise direkt zur Transzendenz, zum Heiligtum fortsetzt. Die Wissenschaft in allen möglichen Formen, konnte den beiden kein richtiges Werkzeug anbieten, um den Menschen und sich selbst zu verstehen. Sie haben die Religion auf ihre eigene Weise gebraucht und versuchten sie in ihr System einzureihen. Nach einem langen Weg des Suchens stellt sich das Verlangen nach der Transzendenz ein, und sie löst die Probleme, welche die Philosophie ohne sie nicht lösen könnte. Einen anderen Weg zur Position Gottes in der Philosophie können wir bei P. Ricoeur finden.

${ }^{12}$ B. Klun, Apologija šibkega krščanstva, in: G. Vattimo, Mislim, da verujem, Ljubljana 2004, S. 11.

${ }^{13}$ G. Vattimo, Glauben - Philosophieren, Stuttgart 1997, S. 68.

${ }^{14}$ Ebd., S. 69. 


\section{Vom Glauben zum Agnostizismus und zurück}

Ricoeur darf man nicht zu denjenigen zeitgenössischen Denkern zählen, die ihre religiöse Überzeugung verheimlicht hätten. Er deklarierte sich durchwegs als Christ und er lebte auch als Christ. Auch in seinem Frühwerk hat er immer eine theologische Überlegung mit eingeschlossen. In seinen späten Werken aber versucht er einen agnostischen Ausgangspunkt anzunehmen, wo er auf dem Untersuchungsweg auf das Gebiet des Glaubens kommt. Er betont, dass er diesen Ausgangspunkt nur wegen des Interesses des Lesers wählt, selbst aber auf die christliche Prägung nicht verzichtet. Vor den Leser will er keine Argumente stellen, die zur Voraussetzungen den biblischen Glauben hätten. „Sie werden bemerken, dass diese Askese in der Argumentierung, die, ich glaube, meine ganze philosophische Arbeit kennzeichnet, zu einem bestimmten Typus der Philosophie führt, wo die Benennung Gottes tatsächlich abwesend ist und wo die Frage über Gott als philosophische Frage genauso in einem Suspenz bleibt, den man agnostisch benennen könnte ${ }^{15}$. Wenn wir bisher den Weg vom agnostischen Ausgangspunkt zum persönlichen Handeln gegangen sind, ist es bei Ricoeur offensichtlich, dass es sich um den umgekehrten Weg handelt.

Ricoeur lehnt einen zu theologischen Zugang ab, weil er dem biblischen Glauben irgendeine Kryptophilosophie beimisst. Es geht also um eine absichtliche Abstandshaltung, die verhindert, die Begriffe miteinander zu vermischen. Das ist noch besonders bedeutungsvoll, weil sein Denkspektrum so vielfältig ist. Auf dieses Problem wurde er aufmerksam, als er Freud philosophisch zu lesen versuchte und es ihm vorgeworfen wurde, dass er als Nichtfachmann darüber spricht. Darum wollte er einen ähnlichen Vorwurf von der Seite der Theologie vermeiden. Auf dem Gebiet der biblischen Hermeneutik und Theologie kann man das Erbe der Phänomenologie feststellen, die in ihrem Verlangen nach der Wissenschaftlichkeit und Objektivität die eidetische Reduktion bis zum Ende durchzuführen versuchte, noch mehr aber Ricoeurs methodische Konsequenz, die auf der objektiven Nachprüfbarkeit basiert. Doch sein ganzes philosophisches Nachdenken ist von Anfang an mit dem Christentum verbunden und sein Bezug auf die Feststellung Spinozas, dass, je mehr wir von den einzelnen Dingen wissen, desto besser kennen wir Gott ${ }^{16}$, ist ein klarer Beweis dafür, dass es sich um eine methodologische Distanz handelt, die noch mehr seine Lebensverbindung und wissenschaftliche Verbundenheit mit der biblischen Tradition und den Glauben an Jesus Christus

${ }^{15}$ Vgl. P. Ricoeur, Réflexion faite, Paris 1995.

${ }^{16}$ In diesem vollkommen eigenständigen Buch, also ganz am Anfang seiner wissenschaftlichen Karriere, benennt er sich selbst als 'Zuhörer des Wortes'. 
betont. „Wenn er das Etikett ,christlicher Philosoph` zurückweist, weigert sich Ricoeur nicht von seinem „Christentum des Philosophen“ (christianisme de philosophe) zu sprechen, ein Ausdruck, den er von Leon Brunschvicg über Jean Nabert geerbt hat, den man keineswegs für einen christlichen Philosophen oder seinen Ersatz halten könnte“"17.

Bei einer solchen Entfernung des wissenschaftlichen Verfahrens vom Bekenntnis des religiösen Ausgangspunktes, stellt uns diese Haltung, (hier kann man nach Belieben den Begriff 'wissenschaftlich' annehmen), eine neue Frage nach ihrer Möglichkeit und Folgerichtigkeit. Der Vorwurf der Kryptotheologie bleibt eine Wirklichkeit, denn die ganze Hermeneutik Ricoeurs öffnet in der letzten Phase das Feld dem Suchen nach der totalen Bedeutung, die nie endgültig entdeckt werden kann, ist aber im dauernden schöpferischen Prozess die Entdeckung eines poetischen Weges zur Erfüllung der letzten menschlichen Sehnsucht. Das ist aber durchaus im Einklang mit der Kant'schen Suche eines rein menschlichen Weges zu Gott. Gleichzeitig betont er immer wieder das Recht und die Pflicht der Denker, dass sie die Ethik und folgerichtig damit die Gesetzgebung nur auf dem menschlichen Niveau begründen, ohne sich auf bestimmte religiöse Voraussetzungen zu berufen. In der Begründung seines Beharrens auf der Religiosität antwortet er dem deklarierten atheistischen Wissenschaftler Jean-Pierre Changeux, dass die Religionszugehörigkeit vor allem die Zugehörigkeit zu einer Tradition ist. Die gibt die Fähigkeit der Sprache, des Verstehens und der Kommunikation und gleichzeitig auch das Bewusstsein, dass es sich nur um einen Weg handelt, der von den anderen verschieden ist. Erst im Rahmen dieser Sprache und Tradition kann man sein eigenes Selbstbewusstsein bilden ${ }^{18}$. Das Akzeptieren des Ausgangspunktes seiner Hermeneutik, dass sich das Subjekt nur in dem Maße versteht, in dem es sich außer sich findet, in den Augen des Anderen, in seinen Schöpfungen, in der Tradition und in der ganzen Gedankenumwelt, ist der notwendige Grund jeder ethischen Haltung des Einzelnen, der komplette Hintergrund der Herstellung des Selbstbewusstseins. Die Schaffenskraft und das Wachsen des eigenen

${ }^{17}$ F. X. Amherdt, Introduction, in P. Ricoeur, L’herméneutique biblique, Paris 2001, S. 14.

Ebenda führt er auch die Behauptung an, die Ricoeur für Panorama 1999 gegeben hatte und wo er behauptet, dass er Gläubiger, Christ protestantischen Glaubensbekenntnisses ist, der sich immer bemüht hatte, eine nötige Distanz zwischen dem Glauben und seinem philosophischen Weg zu behalten.

${ }^{18}$ Vgl. J. P. Changeux und P. Ricoeur, Ce qui nous fait penser. La nature et la règle, Paris 1998. 
Selbstbewusstseins sind nur dort möglich, wo vollkommen neue Begriffe gebildet werden können. Das setzt aber eine Transzendenz voraus ${ }^{19}$.

Die Leitlinie, die sich durch das ganze philosophische Denken Ricoeurs zeigt, ist die Anerkennung der Grenzen. Zu dieser Demut der Philosophie trägt eben die Untersuchung der theologischen Fragen bei. „Diese Philosophie der Grenzen öffnet den Raum der Fähigkeit, das Wort, das von einem Anderen kommt, anzunehmen“'20. Diese Grenzen wurden seiner Ansicht nach mit den sog. modernen Meistern des Zweifels aufgezeigt, zu denen er Nietzsche, Marx und Freud zählt, und sie zerschlagen in erster Linie das selbstaufstellende Subjekt. Wenn sie in ihren Grundgegebenheiten als Philosophen des Atheismus gelten, sind eben sie diejenigen, die für Ricoeur eine neue Möglichkeit des Zutritts zum theologischen Denken öffnen, vor allem aber zeigen sie auf eine klare Forderung nach dem ,zerschlagenen cogito', das nur aus dem Empfangen und dem Dialog lebt. Man kann nicht sagen, dass es sich hier so wie bei Ferry oder Vattimo um einen positiven Prozess der Säkularisierung handelt, es geht vielmehr um das Auslösen des persönlichen hermeneutischen Bewusstseins. Ricoeur würde mit Kant sagen, dass das alles zum Denken gibt. Sicher gab aber die Säkularisierung den beiden Denkern einiges zu denken, dass sie sich auf den Weg des Suchens machten.

Ricoeurs Verbundenheit mit der Hermeneutik zeigt sich auch durch das Suchen eines breiteren Feldes, wo der Einzelne neue Bedeutungen bilden kann. Man wird immer in eine bestimmte Umgebung hineingeboren und so kann man sich seiner Selbst nicht bewusst werden, ohne sich mit der Bedeutung zu identifizieren, die zuerst von Außerhalb auf Einen herankommt ${ }^{21}$. Dieser Grundgedanke leitete ihn auch bei der Forschung nach der Hermeneutik der Symbolen in Symbolik des Bösen. In der Erörterung des religiösen Erbes des abendländischen Menschen verweist er auf zwei Quellen. Die erste ist die griechische Philosophie, die uns durch das Fragen nach dem Seienden das Denken lehrte 22 . Ihr zur Seite erscheint das Judentum als der erste Andere dieser Philosophie, der immer auch ihr Nächster

${ }^{19}$ Dazu können wir aber auch eine versteckte Meinung hinzufügen, dass jeder, der die Welt der Religion nicht annimmt, keinen richtigen und ganzheitlichen Zutritt zu dem Feld hat, wo der schöpferischen Sprache freier Gang gegeben wird.

${ }^{20}$ A. Thomasset, Paul Ricoeur Une poétique de la morale, Leuven 1996, S. 233.

${ }^{21}$ Vgl. P. Ricoeur, Le conflit des interprétation, Paris 1969.

${ }^{22}$ Vgl. P. Ricoeur, Philosophie de la volonté, Paris 1988, S. 183. "Es wird nicht die Überzeugung verschwinden, dass wir für die Philosophie mit den Griechen geboren sind und dass wir, so wie die Philosophen, den Juden vor den Hindu und Chinesen begegneten“. Darum werden wir in seinem Gedanken immer die Beschäftigung mit unserem Erbe finden, das keinen Pol vergessen darf, weder den griechischen noch den jüdischen. 
bleibt. „Die Begegnung der jüdischen Quelle mit dem griechischen Original ist die grundlegende und begründende Kreuzung für unsere Kultur“²3.

Darum hat für ihn eine Überlegung im Rahmen dieses philosophischen Erbes, das neben den philosophischen Wurzeln des Griechentums den zweiten Pol, das jüdische Erbe, das auf dem Religiösen basiert, nicht akzeptieren würde, keine Möglichkeit, die ganze Realität unserer Zivilisation umzufangen.

Wenn also die Philosophie nicht ohne Quellen und Voraussetzungen ist, die sie berücksichtigen und die sie sich in jedem Augenblick auch vor Augen halten muss, kann sie nicht in einer Totalität an sich selbst geschlossen sein. Die ständige Ablehnung Hegels, dem er sich zuerst durch die Kraft seiner Analyse verführen lässt, ist ein ständiges Thema seiner Debatte, besonders wenn es um das Böse und Leiden geht. Die Versuche der Theodizee, die Fragen des Bösen zu umfassen und sie entsprechend zu definieren, scheiterten immer. Darum bleibt bei Ricoeur Platz für die Symbolik des Bösen, die immer zu denken gibt. Das ist aber schon die Domäne des Religiösen, denn es reicht in die Sphäre des ,Sinnüberschusses' (surplus de sens). Diese Symbolik, die ihn in die Richtung der Hermeneutik führt, bringt immer die Zerschlagung aller Versuche, den ganzen Inhalt und Sinn zu umfassen, mit sich. Darum kann man verstehen, warum seine Philosophie die Theologie so meidet, denn im diesem Fall könnte sie den Sinn ausschöpfen und das Symbol, das zu denken geben soll, schließen, oder aus einer lebendigen Metapher eine tote machen, die keine neue Deutung mehr bringen kann.

In seiner Kritik an Hegel kehrt er zu Kant zurück, um so einen Platz für die eigene Erörterung zu machen. In seinem nach-hegelianischen Zugang zu Kant versucht er der Hoffnung Platz zu machen, die er als klare Folge der Frage, „was sollen wir tun“, sieht. In der Abhandlung Freiheit durch die Hoffnung ${ }^{24}$ schlägt er seinen Weg vor, nachdem er die Möglichkeit eines Philosophierens unter Ausschluss dessen, an was wir glauben forderte und auch den Zugang zur Philosophie als der Magd der Theologie zurückgewiesen hat. „Zwischen der Abstinenz und Kapitulation

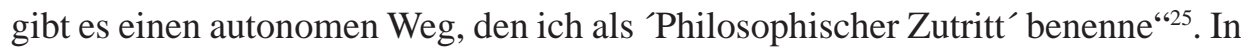
diesem Zutritt deutet er einen Weg an, auf dem man sicher gehen kann, ohne sich in einem der oben erwähnten Extreme zu verirren. Er findet diese Möglichkeit in der Hoffnung. Kants Fragen danach, was man tun soll, seine Moral also, ist keine Lehre, um das Glück zu erlangen, sondern eine Anleitung, wie man dieses Glücks würdig wird. In diesem Fragen nach dem Menschen, dem in der Hoffnung ein Platz

\footnotetext{
${ }^{23}$ Ebd., S. 183.

${ }^{24}$ Vgl. P. Ricoeur, Le conflit des interprétation, Paris 1969.

${ }^{25}$ Ebd., S. 394.
} 


\section{The Person and the Challenges \\ 140 Volume 1 (2011) Number 1}

geöffnet wird, geht es nicht um eine Erweiterung des Wissens oder der Erkenntnis, sondern um eine Annäherung an das, was das Gemeinsame der Philosophie und Theologie ist: 'nur die menschliche Freiheit'. Darum ist das Reden über die Grenzen des Menschen und seine Nicht-Originalität kein Selbstziel, sondern der Weg zur Hoffnung. Dieser Weg ist für ihn der philosophische Zutritt zu den religiösen Fragen. Eben der Begriff der Hoffnung reicht dorthin, wohin, wie er selbst sagt, die sog. ,Chiffren der Transzendenz' reichen würden. Die Forderung des philosophischen Zutritts zur religiösen Offenheit des Menschen, der sein Fundament in der Hoffnung findet, ist ein klares Zeichen dafür, wo wir den Grund für die Notwendigkeit einer entsprechenden Distanz bei der Erörterung Gottes suchen müssen. Sein gesamter hermeneutischer Weg und sein Befassen mit dem Text, mit der Metapher und schließlich auch mit der Ideologie und Utopie, sind eine Suche nach der Möglichkeit, den Menschen für das Transzendente zu öffnen. Mit ihm zusammen können wir uns fragen: „Stellt die Dechiffrierung dieser Chiffre des Transzendenten nicht das vollkommene Modell der Philosophie der Transzendenz, die gleichzeitig auch Poetik wäre, dar?“26.

Die Poetik, die in der Ethik ihr Echo und in der Hoffnung ihren Regulator findet, kann so als Schlüssel zum richtigen Verständnis seiner Überlegungen dienen. Es handelt sich um einen Weg, der zwischen einer religiösen Abstinenz und einem klaren Wunsch nach dem Endbild des hermeneutischen Feldes in der Tradition verläuft, das sich dem Gottesbegriff nicht verschließt. Die Poetik wird als eine Schaffenskraft verstanden, die im Konflikt zwischen einem rationalen Agnostizismus und dem gewollten Aspekt der Welt, der nach dem Licht Gottes ruft, auftritt.

Interessant ist auch die Behauptung, die er in einem seiner letzten Interviews für den Belgischen Rundfunk anführt ${ }^{27}$. Er kehrt zu dem immer anwesenden aber nie klar vorgestellten Philosophen zurück, der selbst viele Zweifel über seine Gläubigkeit und Ungläubigkeit hatte. Spinoza bleibt als diskreter Hintergrund seiner Gedanken. In diesem Gespräch kehrt er wieder zu Spinoza zurück, natürlich im Sinne der Ethik, der ihm eben deswegen entspricht, weil er lediglich über die Moral hinausgeht. Er sagt, dass es um den Weg der Philosophie „in die Totalität des Kosmos geht. Es ist nicht wichtig, ob diese Totalität Natur, Gott oder Pantheismus heißt; das, was zählt, ist die Tatsache, dass wir Teil eines großen Ganzen sind (d'un grand tout). Der Weg in diese Ganzheit, die wir am Anfang noch nicht kennen, erkennen wir aber als Anteil am Ende, führt uns

\footnotetext{
${ }^{26}$ P. Ricoeur, Réflexion faite, Paris 1995, S. 25.

${ }^{27}$ Vgl. P. Ricoeur, L'unique et le singulier, Liège 1999.
} 
zur Freiheit““28. Die Berufung auf die Anfänge, die Platons Eros oder Leibnitz ,appetitio“ versinnbildlichen, ist am besten in conatus eingeschlossen, den er eben bei Spinoza findet und die innere Kraft des Seienden bezeichnet. Wir erkennen gleich beim Ausgangspunkt, den er voraussetzt, bei jener elementaren Leidenschaftlichkeit, die aus diesem absoluten Anfang herausgeht, die Gefahr des Pantheismus. Ricoeur selbst ist sich dessen bewusst und im selben Interview, wie er schon vorher in Das Selbst als ein Anderer ${ }^{29}$ die Möglichkeit des Pantheismus widerruft, als ihn der Interviewer wegen eines Zitats aus Spinoza danach fragte, antwortete er: „Nein. Erstens, weil ich nicht sicher bin, dass es um Pantheismus geht, und endlich, weil mich hier die Frage, ob es um Pantheismus oder Modernismus geht, nicht interessiert. Aber Tatsache ist, dass es eine Ethik sein sollte, genauer gesagt, eine von der Moral unterschiedliche Sache. Die Moral, das ist eine Obligation, sie ist ein Verbot, eine Bestrafung, was dringend seinen Platz hat, im Grunde wegen der Gewalt [...]. Doch es gibt eine Sache, die über die Pflicht hinausschreitet, das ist die Ethik“ ${ }^{\text {“30. Das }}$ grundlegende Wiederauffinden und Selbstverständnis des Menschen auf dem Gebiet der Ethik schreibt er eben dem Begriff des Lebens in conatus zu, so wie das auch Spinoza definiert. Es geht wirklich um die Totalität, die uns umkreist und herstellt. Sie kommt so im Begriff des Charakters vor, den er in seiner These vom Gewollten und Ungewollten bearbeitet, wie auch beim Libido, das er beim Lesen von Freud findet. Doch zu den beiden Begriffen, conatus und libido, stellt er auch telos hinzu, im Sinne Aristoteles, erstens als einen unerreichbaren Horizont unseres Wollens, den er Glück nennt, und zweitens als Prinzip überhaupt jeder Therapie, das er einfach als das teleologische Prinzip der psychoanalytischen Heilung nennt. Gleichzeitig zeigt er uns eine Richtung der Überlegung, die in der Architektur des Subjekts sowohl die ,In-der-WeltSein' im Sinne Heideggers als auch die Offenheit und die Aufhebung im Sinne Hegels zu berücksichtigen versucht: „das ist Spinozas Lektion: zuerst finden wir uns als Sklaven, wir verstehen unsere Sklaverei, dann finden wir uns als Freie in der verstandenen Notwendigkeit wieder. Ethik (es ist Spinozas Werk gemeint) ist das erste Modell dieser Askese, das die Libido, den Willen zur Macht, den

\footnotetext{
${ }^{28}$ Ebd., S. 44.

${ }^{29}$ An dieser Stelle findet er aus dem heideggerianischen Gedanken im spinozistischen Ausdruck conatus eine entsprechende Antwort auf das Verlangen nach dem Aufstellen der Ypseität. Er sagt, dass für ihn nicht die Frage wichtig ist, ob es um Pantheismus oder sogar Atheismus geht; wesentlich ist seine Behauptung, dass es um das Wesen geht, das der Definition nach „essentia actuosa“ ist. Hier treffen sich nach seinen Worten so Spinoza wie der Hl. Paulus, der behauptet, dass wir in Gott leben, uns bewegen und sind.
}

${ }^{30}$ Ebd., S. 44. 
Imperialismus der herrschenden Klasse übertreffen soll. Aber umgekehrt, fehlt in dieser Disziplin des Realen, dieser Askese des Notwendigen die Gnade der Imagination, die Erscheinung des Möglichen? Und diese Gnade der Imagination - hat sie nichts Gemeinsames mit dem Wort als Offenbarung?“31.

\section{Ist die religiöse Erörterung der philosophischen Erörterung nicht würdig?}

Es stört uns Ricoeurs Berufung auf einen bestimmten philosophischen Agnostizismus nicht, weil wir uns bewusst sind, dass es sich bei ihm durch und durch um die Suche einer wahren Antwort auf die Frage nach dem Wort, das groß oder klein geschrieben werden kann, gleichgültig ob es um Gott oder eine neue schöpferische Bedeutung des hermeneutischen Weges geht, handelt. Es geht nicht um die Rechtfertigung des eigenen Lebens, worauf man bei Vattimo schließen könnte, oder um einen notwendigen philosophischen Ausgangspunkt wie bei Ferry. Ricoeur sucht dauernd einen ehrlichen hermeneutischen Weg, wo Gott einen Fortschritt im Verstehen bedeuten würde. Dasselbe könnte man auch für die anderen zwei Philosophen behaupten, nur dass es bei ihnen eine Notwendigkeit ist, obwohl sich Vattimo davor ausdrücklich wehrt ${ }^{32}$, bei Ricoeur aber eine Möglichkeit, die er nach den phänomenologischen Untersuchungen im Menschen und in seinem Erbe findet. Diese kann, seiner Meinung nach, nur durch eine konsequente Hochachtung der Ethik jedes Einzelnen und mit der Aufmerksamkeit zum Fragmentarischen, in dem sich das Ganze widerspiegelt, gefunden werden. Sein Agnostizismus zeigt sich immer mehr nur als methodologischer philosophischer Zutritt. Die philosophische Erörterung religiöser Fragen muss dringend nur daraus wählen, was aus dem Menschen selbst gefolgert wird. Nichts Offenbartes oder dogmatisch Vorgeschriebenes. Das würden auch die beiden anderen unterschreiben, aber erst nach einem stillen Eingeständnis, dass es ohne Transzendenz nicht geht. Die biblische Hermeneutik kann dem Subjekt beim Selbstverständnis nur helfen. Diese Hilfe ist für ihn grundlegend und entscheidend. Ohne Offenheit dem offenbarten Gott gegenüber, der den ganzen Kanon und nicht nur den menschgewordenen Gott einschließt, bleibt das

${ }^{31}$ P. Ricoeur, De l'interprétation, Paris 1965, S. 44.

32 Vgl. G. Vattimo, Glauben - Philosophieren, Stuttgart 1997, S. 72.

„Ich denke an Dietrich Bonhöffers Idee eines mündigen Christentums, das sich seiner Meinung nach nicht mehr an Gott als den höchsten deus ex machina und Löser aller Probleme und Konflikte zu wenden hätte; ....“. 
Subjekt geschlossen für den Grund, der ihm die Einordnung der schöpferischen Gefundenheit in den Rahmen des Daseins als solches ermöglicht.

Ricoeurs Antwort auf die vorher gestellte Frage ist nicht eindeutig. Sein Agnostizismus als Ausgangspunkt ermöglicht diese Grundspannung, die als einziges Schaffensfeld für neue Bedeutungen für den hermeneutischen Zutritt ist, die er intendiert. Die Entfernung von cogito, von eigener Eingenommenheit, löst die grundlegende Fähigkeit des Subjekts aus - die Kreativität. Erst in diesem poetischen Milieu ist Platz für Freiheit und Selbstbewusstsein. Man könnte Ferrys Forderung der horizontalen Transzendenz benutzen, doch Ricoeur schließt auch die vertikale nicht aus, die der horizontalen Transzendenz das Öffnen des horizontalen Schaffens ermöglicht. Die Annahme der Offenheit auf der Ebene der Transzendenz, die über das bloß Menschliche, Irdische hinausgeht, ist nämlich die Bedingung des Ethischen, das der Weg zum Moralischen, zur Sittlichkeit ist. Die Pflicht, die sich mir entgegenstellt, sei es von außen (Gesetze, Vorschriften), oder aus mir selbst (Versprechungen, Pläne, Erwartungen), kann erst dann verantwortungsvoll angenommen werden, wenn man ein klar gestelltes Selbstbild hat. Wissenschaftlicher Agnostizismus ist vor allem der Anfang einer unbeschwerten Ethik, die für das Grundbedürfnis nach der Existenz im Selbstverständnis Lösungen liefert, aber dies nie lösen wird.

Im Gespräch mit dem erwähnten Wissenschaftler, der sich vor allem mit der Untersuchung des menschlichen Gehirns beschäftigt, mit dem Akademiker JeanPierre Changeux, nimmt er immer mehr die Stellung eines Menschen ein, der das Christentum in seiner ganzen Breite aufnimmt, vor allem aber mit seiner Verantwortlichkeit für die Ethik und Lebenssinngebung. Am Ende seines Lebens verbrachte er eine Zeit in der christlichen ökumenischen Gemeinschaft des Bruders Roger in Taizé. In der Tageszeitung ,La croix’ fragt er sich: „Was suchte ich in Taizé? Ich würde sagen, es ging um ein Experiment mit dem, an was ich im innersten glaube: zu wissen, dass der Glaube mit dem Guten zu tun hat. Die christlichen Überlieferungen haben das ein bisschen vergessen. Es geht um eine Not, um eine Gefangenheit in Schuld und Böse. Ich unterschätze dieses Problem nicht, das mich seit mehreren Jahrzehnten hindurch beschäftigte. Aber ich muss überprüfen, dass, auch wenn das Böse noch so radikal sein kann, es nie so tief ist, wie das Gute. Und wenn der Glaube einen Sinn hat, so ist er darin, dass er die Tiefe des menschlichen Guten freilegt, dass er es dort aufsucht, wo es total begraben ist“. Wenn wir noch die Worte dazugeben, die er im Gespräch mit Changeux sagte: „Das Gute muss man befreien, es wurde zum Gefangenen der freien Handlung - seinen größten Absurd zeigte die Freiheit in Auschwitz“"33,

\footnotetext{
${ }^{33}$ J. P. Changeux und P. Ricoeur, Ce qui nous fait penser. La nature et la règle, S. 253.
} 
können wir klar sehen, was sein Ausgangspunkt war, der ihn in seiner ganzen Forschungsarbeit begleitete: das wiedergefundene Subjekt, zu dem die ethische Ebene hinzukommt, als notwendige Schlussfolgerung, ohne die es diesen Weg nicht geben könnte.

Diese ,mini Ethik', wie er sie selbst benennt, ist keineswegs zweitrangig, oder nur die Folge des hermeneutischen Weges, sie ist der Grundzug seiner Erörterung. Hier muss es immer Platz für die Freiheit geben. Darum ist auch für den Gläubigen einzig der agnostische Ausgangspunkt Gewähr für die wahre Kreativität. Erst mit der Offenheit für verschiedene Bedeutungen, mit dem Austreten aus der Tradition, die den Menschen an der Natur orientiert, vermag der Gläubige eine bewusste und schöpferische Haltung vor Gott einzunehmen. Andererseits muss sich der agnostisch gerichtete Wissenschaftler bemühen, seinen Ausgangspunkt und seine Erwartungen zu vertiefen, um seinem Gesichtspunkt eine genügende Distanz zu sichern, die den Weg zur Kreativität öffnet, besonders, da Kritikvermögen und Schaffenskraft die Notwendigkeit jeder wissenschaftlichen Betrachtung sind. Die ist nur in Offenheit neuen Bedeutungen gegenüber möglich. Wenn auch die agnostische Haltung als wissenschaftlicher Ausgangspunkt vorzuziehen ist, darf sie nicht schon in Voraus als einziges Ziel intendiert werden, da sie das Feld den möglichen Bedeutungen vor den Herausforderungen des Transzendenten und gleichzeitig den eingeborenen hermeneutischen verschließt, und somit auch dem Wissenschaftler irgendwie seine Freiheit nimmt.

\section{Zum Schluss}

Es ist verständlich, dass Ricoeur nach seinem philosophischen Weg im Theismus und in einer klaren Entscheidung für das Christentum landete, denn er hat seinen Glauben auch immer gelebt. Im Beispiel, das er Changeux gibt, sagt er, wenn auf einer großen Kugel, die auch das Bild der Erde sein könnte, jeder den Weg zum Anderen nur auf der Oberfläche suchen würde, könnte man lange nicht zueinander finden. Würde aber jeder an seiner Stelle in die Tiefe forschen, würde jeder der Mitte immer näher kommen und somit auch einander. Ricoeur weist nichts im Voraus zurück, was bei Vattimo und bei seiner Abneigung zu den Institutionen offensichtlich ist, sondern entdeckt versteckte Bedeutungen, die ihm auf dem Weg zu dem Anderen und damit auch zu sich selbst behilflich sind. So weicht er der Kritik Vattimos aus, wo dieser (Vattimo) Ricoeurs Treue zu Husserls Forderung nach der Philosophie als strenger Wissenschaft nicht versteht: „Ricoeur, der sich auf 'Zeit und Erzählung' konzentriert, hat vielleicht 
diese Notwendigkeit eines Neu-Denkens der Geschichte erfasst, auch wenn er es ein weiteres Mal auf der Ebene einer strukturellen Beschreibung anstatt in einem radikalen Entwurf der Hermeneutik als Moment der Seinsgeschichte einzulösen scheint ${ }^{\star 34}$. Eben die Konsequenz der Methode ermöglicht ihm, den Weg dem neuen Verstehen des Seins zu öffnen und das auf der Ebene des Poetischen, das sich ihm in großem Maße eben in der Metaphorik der biblischen Überlieferung ,offenbart‘.

Es ist notwendig zu erwähnen, dass seine Auseinandersetzung mit der biblischen Hermeneutik immer auch streng wissenschaftlich ist. Sie ist noch besonders durch die analytische und strukturalistische Methode gekennzeichnet, die, seiner Meinung nach, den hermeneutischen Kreis der Gefangenschaft im Relativismus des Subjektivismus öffnet. Doch er sucht in allen biblischen Texten die Antwort auf die ursprüngliche Sehnsucht, die dem konkreten Menschen einbeschrieben ist. Darum können wir jetzt seine Begründung des Agnostizismus verstehen. Der Leser ist derjenige, der das Zentrum jeder Forschung und Erörterung ist, auch auf dem Gebiet der Offenbarung. Diese endliche Vorauskennzeichnung des Subjekts mit dem Christentum, die einen immer überholt, ermöglicht ihm, sich selbst und seine Voraussetzungen aufzunehmen und öffnet ihn zur Schaffenskraft, die er in den schöpferischen hermeneutischen Prozess der gesamten Menschheit dazugeben wird.

Das grundlegende hermeneutische Prinzip ist die Notwendigkeit, sich selbst zu entfernen, was auch der Begriff der Transzendenz bei Ferry voraussieht. Doch Ferry weist die klassische theologische Sprache zurück, und sucht sie immer mehr in der alltäglichen Erfahrung des Einzelnen. Dabei vergisst er jedoch, dass die biblische Sprache eine Auswahl der am meisten offenbarenden Erfahrungen des Einzelnen und des Volkes ist. Für Ricoeur ist die endgültige und die größte schöpferische Entfernung, die gleichzeitig auch Beweger aller Wissenschaft ist, eben die religiöse Sprache. Die entstand gerade mit der Offenheit in das Transzendente der Bedeutung. Die Mythen und Symbolik, die grundlegende religiöse Sprache, zeigen deutlich dem Subjekt, das sich von ihnen her versteht, dass es begrenzt aber gleichzeitig imstande ist, diese Grenzen zu überschreiten. Das Annehmen der Grenzen und Voraussetzungen ist endlich eine der Grundbedingungen der Wissenschaft als solcher, die auch nach seinem Maß des Menschen wäre. Er ist nämlich Alpha und Omega aller wissenschaftlichen Bestrebungen. Doch er würde nie forschen, wenn er nicht etwas davon erwarten würde. Eben in der Religion können wir beides finden. Der Mensch fand sich vor dem Unbegreiflichen, darum ,erfand‘ er die Sprache, die sowohl seine

\footnotetext{
${ }^{34}$ G. Vattimo, Abschied, Wien 2003, S. 75.
} 


\section{The Person and the Challenges \\ 146 Volume 1 (2011) Number 1}

Beschränktheit (Unfähigkeit, das in vollem Maße auszudrücken) als auch das Staunen, dass er das überhaupt wahrnimmt, mit einschließt. In der ganzen Religion ist eine Bedeutung versteckt, die schon von sich aus die Transzendenz bringt. Vattimo und Ferry sind erst auf der Suche nach dieser Transzendenz. Sicher ist aber ihr Weg eine neue Art und Weise, um eine empfindliche Sprache für die religiöse Erfahrung schaffen zu können. Mit Vattimo zusammen können wir sagen: „Dass der philosophische Kern des gesamten hier entwickelten Diskurses die Hermeneutik ist, die Philosophie der Interpretation, zeigt seine unerschütterliche Treue gegenüber der im zweifachen Sinn verstandenen Idee der Gnade: als Geschenk, das von einem Anderen herkommt, und als Antwort, die, indem sie die Gabe annimmt, davon untrennbar auch die ureigene Wahrheit dessen, der sie empfängt, zum Ausdruck bringt“‘35.

Trotz des hermeneutischen Eifers könnte man bei Vattimos und Ferrys Versuch schwer sagen, dass es bei ihnen um ein ähnliches Verstehen des Menschen geht wie bei Ricoeur. Sie selbst behaupten, dass sie den Menschen befreien und gleichzeitig erhöhen wollen. Der Mensch braucht die Transzendenz, die in ihm selbst ist. Wenn die, laut Ricoeur, gegeben ist, in Symbolen, Metaphern und ähnlichem offenbart ist, dann ist das für sie nur ein Notausgang. Da sie in ihren Erörterungen nicht anders können, weil sie es einsehen, dass der Mensch die Entfernung von sich selbst braucht, schreiben sie ihm das einfach selbst zu, seiner eigenen Fähigkeit. Die Beziehung zu dem anderen vermenschlicht sich von sich selbst. In der Vermenschlichung Gottes, wie das Ferry versteht, ist das Subjekt vor der Gewalt des Äußeren geschützt. Es selbst wird sein Herr und gleichzeitig hat er kein Werkzeug, um den Weg aus dieser Einsamkeit zu finden. Der andere spricht ihn nicht als Heiligtum, sondern nur als Bedürfnis an. Wenn wir den schon gebrauchten Vergleich der Kugel benutzen, können wir sagen, dass der Mensch auf diese Weise nicht gezwungen ist, in die Tiefe zu gehen. Er wird bei sich selbst bleiben und sich mit sich selbst beschäftigen. Alle Gründe, die Ferry für die Transzendenz anführt, sind nur die Folge der Beschäftigung mit sich selbst und verweisen nicht darauf, dass der Mensch fähig wäre, dem Neuen, Offenbarten zuzuhören. Wenn der Mensch die Quelle der Transzendenz ist, kann leicht passieren, dass man die Empfindlichkeit für die Transzendenz verliert. Der Andere ist wirklich der andere, doch schnell kann er nur zum Mittel werden, das man ganz eigenwillig verstehen kann. Hier ist Levinas Forderung am Platze, Ethik vor die Ontologie zu stellen, da die Verbundenheit mit dem Anderen ursprünglicher ist als das Denken über sich selbst. Wie wird

\footnotetext{
${ }^{35}$ G. Vattimo, Glauben - Philosophieren, S. 113f.
} 
die Säkularisierung, wie sie Vattimo versteht, ihre Aufgabe der Schwächung durchführen können, wenn sie den Menschen allein wie einen Gott stehen lassen würde, der wie ein Narziss auf sich selbst starren wird? Freilich ist es viel leichter, sich selbst zu akzeptieren, wenn man sich schon im Voraus für göttlich hält, doch das führt nicht zum Wachsen und noch weniger dazu, dass man in der Anrede des Anderen, den Anderen als sich selbst sehen könnte. Für Ricoeur ist der Weg des Konflikts die Grundlage der Interpretation und damit auch das Verstehen von sich selbst, für Vattimo ist die Konfliktträchtigkeit der Welt nur Notwendigkeit für die Rückkehr des schon erlebten Verständnisses und zusätzlich, wie es seinem Bedürfnis entspricht, zur Hoffnung, dass er trotz allem glaubt. Dieser zurückerhaltene Glaube bringt in seine Welt nichts Neues, noch weniger nimmt er eine neue ethische Verbundenheit in Anspruch. Die Hoffnung, die Vattimo auf Grund des Glaubens voraussetzt, ist nur die Folge unausgefüllter Wünsche hier und jetzt, bei Ricoeur aber, immer wieder die Hoffnung auf schöpferische Beziehung mit dem sich Offenbarenden. Eine Offenbarung, die nur an das Ereignis, das wir erleben, gebunden wäre und nicht das ganze Erbe und alle Erwartungen, die uns gegeben wurden, berücksichtigen würde, bliebe ein ewiges Zurückkehren des ewig Bekannten und wir würden dieses Zurückkehren nicht einmal erkennen. Die Konfliktträchtigkeit des agnostischen Ausgangspunktes in der philosophischen Erörterung führt zur Suche nach der Quelle des Konflikts. Es zeigt sich von selbst, dass der Ursprung nicht in uns selbst liegt, sondern dass er über uns hinausgeht. Gleichzeitig wird der Anfang der Antwort in der Tradition gegeben und fordert die Menschwerdung in jedem Einzelnen, denn sie muss ihm seinen eigenen Daseinshorizont öffnen. Die Schwächung im Sinne Vattimos kann in Wirklichkeit nur eine Frage, aber keine Antwort sein. Im Begriff der Wahrheit wird etwas Festes verstanden. Diese Erwartung zwingt den Menschen zur Kreativität. Darum ist das Poetische der Grund für das Verständnis, sowohl von sich selbst, von der Welt, wie auch von allem, was über sie hinausgeht. Bei der Berufung auf die Hermeneutik könnten so Vattimo wie auch Ferry dem Menschen und seiner Fähigkeit mehr vertrauen, das Andere zu ertragen. Gleichzeitig sollten sie aber auch erlauben, im Anderen die Welt, die uns immer wieder vom Weg abbringt, zu finden und zuzulassen.

Wenn die Hermeneutik vor allem Verständnis ist, dann kann man daraus auch den Mechanismus nicht ausschließen, der diesem Verständnis frische Herausforderungen gibt. Diese können aber nicht aus immer demselben kommen, sondern aus dem, was mich immer wieder unter das Fragezeichen stellt. Vattimos 


\section{The Person and the Challenges \\ 148 Volume 1 (2011) Number 1}

trotziges Verharren darauf, dass sein Zurückkehren keinesfalls auch eine neue ethische Beurteilung des Lebens deutet, verweist deutlich auf die Beschränktheit dieses Versuchs. Das Ereignis in der Beziehung zu dem Anderen ist keine Sache des Verständnisses, sondern einer gelebten Beziehung. Eine Hermeneutik, die diesen Aspekt ausschließt, verliert die Grundladung, die sie immer aufs Neue in den Kreis des Verständnisses treibt. Ricoeur geht im hermeneutischen Text davon aus, dass das Treffen mit der neuen Bedeutung vor allem Mimesis bedeutet, also Nachahmung, die noch immer schöpferisch ist. Das schwache Denken bleibt wirklich kraftlos, denn es lässt den Menschen so, wie er ist.

Ferry versucht die Frage der Beziehung dem anderen gegenüber mit dem Begriff des Heiligen zu lösen: „Wenn wir nicht diese Erfahrung der persönlichen Liebe, als auch die Erfahrung der Gemeinschaft hätten, die öfter gebaut wird und zwar nicht aus sich selbst, sondern aus ,sym-pathie‘, glaube ich, dass wir heute in der säkularisierten und entzauberten Welt keine Erfahrung des Heiligen mehr hätten“36. Es geht um die Fähigkeit, sich dem Anderen zu öffnen, ihn zu erfühlen. Damit sind wir schon bei irgendeiner Offenbarung; der Andere zeigt sich mir als etwas Heiliges, was ich nicht besitzen kann, aber dieses Heilige überredet mich, mich für es zu begeistern, es zu verstehen, es anzusprechen, mich für es zu opfern. Dadurch wird aber der Einzelne in der Regel nicht vergöttlicht, sondern eher erniedrigt, begrenzt, es flößt ihm Schuldgefühle ein. Neben dem Anderen kann ich öfters wie Kain stehen, der nicht die Möglichkeit hat, Gott zu begegnen und dabei eine Geborgenheit zu erleben. Erst dann, wenn er bei dieser Offenheit zu glauben vermag und einen hermeneutischen Hintergrund erleben wird, dass auch er Teil einer sinnvollen Welt und so schon im Voraus heilig ist, würdig ist, dass sich einer für ihn opfert, wird er das als Einladung auf einen selbständigen Weg des schöpferischen Lebens anzunehmen vermögen. Für Ricoeur sind die Erfahrung der Schenkung Christi, ohne Gegenleistung, und das Symbol des Kreuzes die grundlegende Erfahrung, die ihn schon im Voraus ansprechen und ihm schon im Voraus das Gefühl der Gemeinschaft geben. In dieser Vorerfahrung des ,Paradieses‘, das im gebrochenen Bild des menschlichen Lebens vor allem die Erfahrung der Abwesenheit ist, öffnet sich für ihn auch der Raum für die Hoffnung. Küng würde sagen, dass es sich um eine typisch christliche Dimension handelt: „Christlicher Glaube freilich ist ein radikalisiertes Grundvertrauen, insofern ich mich nicht nur auf die Wirklichkeit einlasse, sondern auf den Urgrund, Urhalt und das Urziel aller Wirklichkeit“37. Beide, so Vattimo wie Ferry suchen die Hoffnung, die über die Gefangenheit in einem begrenzten Raum

\footnotetext{
${ }^{36}$ A. Comte-Sponville und L. Ferry, La sagesse des Modernes, S. 445.

${ }^{37}$ H. Küng, Wozu Weltethos?, Freiburg im Breisgau 2002, S. 166.
} 
und einer begrenzten Zeit gehen und gleichzeitig zum Grund des Menschlichen zurückkehren würde; bei Ricoeur ist das so der Grund des Lebens aus dem Glauben wie auch die Verbundenheit zu einer ernsten wissenschaftlichen Arbeit eines Philosophen.

Die Wissenschaft auf einem humanistischen Gebiet lässt keine einfachen Antworten zu, wie man sie in den herkömmlichen philosophischen Handbüchern finden kann: „Ontologisch lässt sich der Tod verstehen als ein Verlassen der einzigen Wirklichkeit, zu der dieses Leben geworden ist, als Rückkehr in den unendlichen Raum der Möglichkeit, als ein 'Heimgehen' in diesem Sinne, da das Selbst ursprünglich von dort gekommen ist“" ${ }^{\star 38}$. Obwohl man auf diese Weise die Angst und Ohnmacht vor dem Tod am leichtesten überwinden würde, ist das für die schöpferische Spannung nur ein Ausweg der 'faulen' hermeneutischen Vernunft. Die Konfliktträchtigkeit bringt Spannung, die das schöpferische Verständnis fordert und gleichzeitig das ganze Leben kennzeichnet. Bei Vattimo und Ferry könnte man schwer so eine Spannung des Konfliktes finden, ebenso keine schöpferische Fähigkeit, welche das Leben ändern würde, natürlich mit dem Glauben an die Suche des Guten. Es stimmt, dass Ricoeur kein ontologisches Bild des postmodernen Verstehens anbietet und gleichzeitig keine genauen moralischen Anweisungen gibt, er öffnet aber die Möglichkeit, dass der Einzelne als Person sich selbst bejaht und an den Anderen glaubt, der sich ihm offenbart, dabei gelingt es ihm aber, sich auch mit dem ewig Anderen zu treffen.

\section{Bibliography}

Amherdt F. X., Introduction, in: P. Ricoeur, L'herméneutique biblique, Paris 2001, Cerf.

Changeux J. P. und P. Ricoeur, Ce qui nous fait penser. La nature et la règle, Paris 1998, Editions Odile Jacob.

Comte-Sponville A. und L. Ferry, La sagesse des Modernes, Paris 1998, Robert Laffont.

Ferry L., L'Homme-Dieu, Paris 1996, Grasset.

Habermas J., Glauben und Wissen, Frankfurt am Main 2001, Suhrkamp.

Klun B., Apologija šibkega krščanstva, in: G. Vattimo. Mislim, da verujem, Ljubljana 2004, KUD Logos.

Küng H., Wozu Weltethos?, Freiburg im Breisgau 2002, Herder.

\footnotetext{
${ }^{38}$ W. Schmid, Mit sich selbst befreundet sein, S. 428.
} 
Ricoeur P., Le conflit des interprétation, Paris 1969, Seuil.

Ricoeur P., De l'interprétation, Paris 1965, Seuil.

Ricoeur P., Histoire et vérité, Paris 1955, Seuil.

Ricoeur P., Philosophie de la volonté, Paris 1988, Aubier.

Ricoeur P., Réflexion faite, Paris 1995, Esprit.

Ricoeur P., Soi-même comme un autre, Paris 1996, Seuil.

Ricoeur P., L'unique et le singulier, Liège 1999, Alice.

Schmid W., Mit sich selbst befreundet sein, Frankfurt am Main 2004, Suhrkamp. Thomasset A., Paul Ricoeur Une poétique de la morale, Leuven 1996, University press.

Vattimo G., Abschied, Wien 2003, Turia + Kant.

Vattimo G., Glauben - Philosophieren, Stuttgart 1997, Reclam. 